\title{
Ansiedad y depresión en pacientes con enfermedad pulmonar obstructiva crónica
}

\section{Anxiety and depression among patients with chronic obstructive pulmonary disease}

\author{
Adalberto Campo-Arias, John Carlos Pedrozo-Pupo, Guillermo Augusto Ceballos-Ospino \\ - Santa Marta (Colombia)
}

DOI: https://doi.org/10.36104/amc.2021.1644

\section{Resumen}

Objetivo: establecer algunas variables asociadas a ansiedad y depresión con importancia clínica en pacientes con EPOC de la consulta externa de Santa Marta, Colombia.

Diseño: estudio analítico transversal.

Marco de referencia: la enfermedad pulmonar obstructiva crónica (EPOC) es un padecimiento crónico que afecta aproximadamente a una de cada diez personas de la población general colombiana. La EPOC con frecuencia se asocia a ansiedad y depresión. Sin embargo, es limitada la información sobre las variables asociadas a ansiedad y depresión en pacientes con EPOC.

Participantes: se incluyeron pacientes adultos con EPOC. Los pacientes se clasificaron según la iniciativa GOLD y la evaluación combinada.

Intervenciones: ninguna.

Mediciones: la ansiedad y la depresión con importancia clínica se evaluaron con las versiones de 10 ítems de las escalas de Zung.

Resultados: participaron 409 pacientes entre 40 y 102 años, media de 73.0 años (DE=10.2 años), $58.7 \%$ de sexo masculino, $88.8 \%$ casado (o en unión libre), $73.6 \%$ residentes en estrato bajo (I, II o III), 92.7\% expuesto a cigarrillo o humo de leña (biomasa), 44.5\% en GOLD 3 o 4, $22.2 \%$ clasificados en la evaluación combinada C o D, $19.1 \%$ presentaron ansiedad con importancia clínica y $27.9 \%$ depresión con importancia clínica. La ansiedad con importancia clínica mostró asociación significativa con la evaluación combinada C o D (OR=2.08; IC95\% 1.19-3 .63) y sexo femenino $(\mathrm{OR}=1.80$; IC95\% 1.10-2.99) y la depresión con importancia clínica mostró relación significativa con sexo femenino $(\mathrm{OR}=2.35$; IC95\% 1.50-3.70) y la evaluación combinada C o D (OR=2.04; IC95\% 1.22-3.42).

Conclusiones: la prevalencia de ansiedad y depresión con importancia clínica es alta en pacientes con EPOC. Ansiedad y depresión se asocian a sexo femenino y a la gravedad de la EPOC. Es necesario evaluar ansiedad y depresión en todos los pacientes con EPOC evaluados en consulta externa, particularmente, en mujeres y en pacientes clasificados C o D en la evaluación combinada. (Acta Med Colomb 2021; 46. DOI: https://doi.org/10.36104/amc.2021.1644).

Palabras clave: ansiedad; depresión; enfermedad pulmonar obstructiva crónica; pacientes ambulatorios; estudios transversales.

\section{Abstract}

Objective: to establish some variables associated with anxiety and depression with clinical importance in patients with COPD from the outpatient clinic in Santa Marta, Colombia.

Design: a cross-sectional analytical study.

Background: chronic obstructive pulmonary disease (COPD) is a chronic disease that affects approximately one in ten people in the general Colombian population. COPD is often associated with anxiety and depression. However, information on the variables associated with anxiety and depression in patients with COPD is limited.

Participants: adult COPD patients were included. The patients were classified according to the GOLD initiative and the combined assessment.
Dr. Adalberto Campo-Arias: Psiquiatra. Especialista en Epidemiología, Magíster en Salud sexual. Profesor Asociado Programa de Medicina; Dr. John Carlos Pedrozo-Pupo: Internista, Neumólogo. Profesor Asistente Programa de Medicina; Dr. Guillermo Augusto CeballosOspino: Psicólogo. Especialista en Gerencia de los Servicios de Salud y Profesor Ocasional Programa de Psicología. Facultad de Ciencias de la Salud, Universidad del Magdalena. Santa Marta (Colombia).

Correspondencia: Dr. Adalberto Campo-Arias. Santa Marta (Colombia)

E-Mail: acampoa@unimagdalena.edu.co Recibido: 13/I/2020 Aceptado: 29/I/2021 
Interventions: none.

Measurements: clinically important anxiety and depression were assessed with the 10-item versions of the Zung scales.

Results: 409 patients between 40 and 102 years of age participated, mean of 73.0 years (SD=10.2 years), $58.7 \%$ male, $88.8 \%$ married (or in free union), $73.6 \%$ residents in low stratum (I, II or III), $92.7 \%$ exposed to cigarettes or wood smoke (biomass), $44.5 \%$ in GOLD 3 or 4, $22.2 \%$ classified in the combined evaluation C or D, $19.1 \%$ presented clinically significant anxiety and $27.9 \%$ clinically significant depression. Clinically important anxiety showed a significant association with the combined assessment $\mathrm{C}$ or $\mathrm{D}(\mathrm{OR}=2.08,95 \% \mathrm{CI} 1.19-3.63)$ and female sex (OR=1.80,95\%CI 1.102.99) and depression with clinical importance showed a significant relationship with female sex $(\mathrm{OR}=2.35,95 \% \mathrm{CI} 1.50-3.70)$ and the combined $\mathrm{C}$ or $\mathrm{D}$ evaluation $(\mathrm{OR}=2.04,95 \% \mathrm{CI}$ 1.22-3.42).

Conclusions: The prevalence of clinically important anxiety and depression is high in COPD patients. Anxiety and depression are associated with female gender and the severity of COPD. Anxiety and depression must be evaluated in all patients with COPD evaluated in an outpatient clinic, particularly in women and in patients classified C or D in the combined evaluation. (Acta Med Colomb 2021; 46. DOI: https://doi.org/10.36104/amc.2021.1644).

Key words: anxiety, depression,chronic obstructive pulmonary disease, outpatients, crosssectional studies.

\section{Introducción}

La enfermedad pulmonar obstructiva crónica (EPOC) es un problema médico crónico, con periodos de remisiones parciales y otros de exacerbaciones, que se caracteriza por reducción en el flujo de aire y aumento en la respuesta inflamatoria; estos cambios en la fisiología respiratoria se traducen en el contex to clínico en disnea, tos y expectoración (1). La EPOC afecta aproximadamente a una de cada diez personas de la población colombiana (2).

La prevalencia de ansiedad y depresión suelen ser altas en pacientes con patologías crónicas como la EPOC (3-8). En un meta-análisis se observó que los pacientes con EPOC presentaron tres veces más probabilidades de presentar depresión que la población general (9).

Por otra parte, estudios transversales muestran que la prevalencia de ansiedad en pacientes con EPOC se encuentra entre $15.2 \%$ y $19.1 \%(10,11)$. En el contexto de Santa Marta, en un estudio transversal se observó que 5.8\% de los pacientes con EPOC refirió el antecedente de depresión; sin embargo, no se informó la presencia de ansiedad (12).

Las investigaciones sugieren que la relación entre ansiedad, depresión y EPOC es bidireccional, la presencia de cada una incrementa la posibilidad de reunir criterios para la otra $(3,13)$. La EPOC deteriora de manera importante la calidad de vida de las personas $(14,15)$, y en presencia de ansiedad y/o depresión, la calidad de vida de los pacientes se deteriora aún más $(4,5,14,16)$. Además, estos pacientes tienen un riesgo significativamente mayor de hospitalización por exacerbaciones de la $\operatorname{EPOC}(17,18)$, así como de estancias hospitalarias más prolongadas, de muerte tras el alta hospitalaria $(13,18)$ y un incremento del riesgo suicida $(19,20)$.

En relación con las variables asociadas a ansiedad y depresión en pacientes con EPOC, se ha observado que el compromiso en la calidad de vida se relaciona directamente con la gravedad clínica, mediada por la presencia de ansiedad y depresión en este grupo de pacientes $(5,14,16$, 21). La relación se mantiene en el rango significativo, aun cuando se controla el consumo de cigarrillo (15), el cual es necesario, ya que los componentes del humo del tabaco pueden modificar la prevalencia de ansiedad y depresión en personas sin EPOC $(22,23)$.

No obstante, en el contexto mundial y nacional, el estudio de las variables asociadas a ansiedad y depresión en pacientes con EPOC se ha centrado, en particular, en la asociación con el deterioro en la calidad de vida sin considerar otras variables que pueden generar confusión $(5,14-16,21)$.

Precisar y ajustar las variables asociadas a ansiedad y depresión amplía el conocimiento en el área. La mejor comprensión de la relación entre ansiedad, depresión y EPOC puede ayudar a la implementación de acciones de detección temprana de casos (6) y a reducir la carga de la comorbilidad y al mejoramiento de la calidad de vida (5, 14, 15).

El objetivo general del presente estudio fue establecer algunas variables asociadas a ansiedad y depresión con importancia clínica en pacientes con EPOC en la consulta externa de neumología de Santa Marta, Colombia.

\section{Material y métodos \\ Diseño y consideraciones éticas}

Se diseñó un estudio observacional analítico transversal que consideró los aspectos éticos de la investigación en las que participan humanos de la Resolución 8430 de 1993 del Ministerio de Salud de Colombia. El proyecto contó con el aval del Comité de Ética en Investigación de la Universidad del Magdalena, Santa Marta, Colombia. Todos los participantes lo hicieron de manera voluntaria después de firma de 
consentimiento informado. Los participantes no recibieron incentivo alguno por la inclusión en el estudio (24).

\section{Participantes}

Se solicitó la participación de adultos que consultaron a tres instituciones de Santa Marta, entre agosto y diciembre de 2019. Se incluyeron adultos mayores de 40 años, con diagnóstico de EPOC, quienes firmaron el consentimiento informado. Se excluyeron mujeres embarazadas y pacientes con deterioro cognitivo que limitara completar las escalas de medición para ansiedad y depresión.

\section{Muestreo}

Se siguió un muestreo no probabilístico de pacientes evaluados de manera consecutiva. Basado en los registros históricos de las instituciones participantes se esperaba contar una muestra de por lo menos 267 personas. Este número se estimó a partir de una prevalencia esperada del $25 \%$ de ansiedad o depresión, margen de error de 5 y nivel de confianza de $95 \%$ (25). Se esperaba un odds ratio $(O R)$ de 3.0 y para ello se necesitaban por lo menos 87 pacientes con ansiedad o depresión y 87 pacientes sin ansiedad o depresión con importancia clínica, con nivel de confianza de $95 \%$ y un poder de $80 \%$ (26). Así mismo, ese tamaño de muestra permitiría, hacer ajustes en las asociaciones con la inclusión hasta de seis variables en bloque, bajo el supuesto que se contarían con no menos de 67 casos de ansiedad y de depresión con importancia clínica, buscando contar con 10 casos por cada posible variable de confusión (27).

\section{Instrumentos}

Se registraron datos sociodemográficos (edad, sexo, estado marital, estrato socioeconómico y ciudad de residencia), hallazgos clínicos como la evaluación combinada y clasificación GOLD y las puntuaciones en las escalas de Zung para ansiedad y depresión.

\section{Clasificación GOLD}

La clasificación GOLD para la EPOC toma el grado de limitación del flujo aéreo que presenta el paciente medido con el flujo espiratorio forzado al minuto (FEV1) y clasifica según el grado de restricción en 1, 2, 3 y 4, de menor a mayor restricción (28). En el presente estudio los pacientes se categorizaron en dos grupos: baja restricción (GOLD 1 y 2) y alta restricción (GOLD 3 y 4 ).

\section{Evaluación combinada}

Es una clasificación más reciente del estadio clínico de la EPOC, implica una evaluación combinada que considera los síntomas del paciente, número de exacerbaciones y gravedad de los síntomas, además de calidad de vida medida con CAT (COPD Assessment Test). Incluye los estadios A, B, C y D. La clasificación A es el estadio de menor gravedad y el D, el de mayor gravedad (27). En la presente investigación los estadios se categorizaron en dos grupos menor gravedad (A y B) y mayor gravedad (C y D).

\section{Escala de Zung para Ansiedad}

Los pacientes diligenciaron la escala breve de Zung para ansiedad. Esta escala consta de diez ítems que explora síntomas ansiosos durante el último mes. Ofrece un patrón de respuesta politómica con cuatro opciones: nunca, a veces, muchas veces o siempre. Estas posibilidades se califican de cero a tres, en consecuencia, las puntuaciones totales posibles están entre cero y treinta. Las puntuaciones mayores a diez se categorizaron como síntomas de ansiedad con importancia clínica. Esta escala ha mostrado aceptable desempeño en algunos indicadores psicométricos en muestras de participantes colombianos (29). En el presente estudio, el alfa de Cronbach fue 0.82 .

\section{Escala de Zung para depresión}

La escala breve de Zung para depresión se compone de diez ítems que exploran síntomas depresivos durante las dos últimas semanas. Ofrece un patrón de respuesta politómica con cuatro opciones de respuesta: nunca, a veces, muchas veces o siempre. Estas respuestas se califican de cero a tres, con puntuaciones totales entre cero y treinta. Las puntuaciones superiores a diez se categorizaron como síntomas depresivos con importancia clínica. Esta escala ha mostrado buen desempeño psicométrico en un estudio colombiano precedente (30). En la presente muestra, el alfa de Cronbach fue 0.78 .

\section{Procedimiento}

Primero, se explicó a los pacientes los objetivos de investigación. Los pacientes que firmaron consentimiento informado completaron las escalas de Zung para ansiedad y depresión que no hacen parte del protocolo habitual de evaluación y seguimiento en la consulta externa de pacientes con EPOC. La inclusión de los pacientes se hizo siguiendo el orden de llegada hasta la fecha límite propuesta.

\section{Análisis estadístico}

El componente descriptivo incluyó la observación de la distribución de los datos. Se estimaron frecuencias y porcentajes para las variables cualitativas y media (M) y desviación estándar (DE), mediana (Me) y rango intercuartílico (RIC) para las variables cuantitativas. No se consideró la posibilidad de valores perdidos dado la recolección prospectiva de la información.

En el análisis bivariado, se tomaron en forma separada ansiedad y depresión con importancia clínica como variables dependientes y las variables demográficas, estadio GOLD y la evaluación combinada se tomaron como variables independientes todas en forma dicotómica. Se calcularon OR con intervalos de confianza de 95\% (IC95\%).

En el análisis multivariado, se ajustaron por las variables que en el análisis bivariado mostraron valores de probabili- 
Tabla 1. Descripción demográfica de la población.

\begin{tabular}{|l|c|c|}
\hline \multicolumn{1}{|c|}{ Variable } & Frecuencia & $\%$ \\
\hline Edad (años) & & \\
44-64 65 o más & 318 & 22.2 \\
& & 77.8 \\
\hline Sexo & 169 & \\
Femenino & 240 & 41.3 \\
Masculino & & 58.7 \\
\hline Estado marital & 254 & 88.8 \\
Casado (y unión libre) & 46 & 11.2 \\
Soltero & & \\
\hline Estrato & 301 & 73.6 \\
Bajo (I, II o III) & 108 & 26.4 \\
Alto (IV, V o VI) & & \\
\hline Historia de consumo de cigarrillo & 355 & 86.8 \\
Sí & 54 & 13.2 \\
No & & \\
\hline
\end{tabular}

Tabla 2. Características clínicas de la población.

\begin{tabular}{|l|c|c|}
\hline \multicolumn{1}{|c|}{ Variable } & Frecuencia & $\%$ \\
\hline GOLD & 182 & \\
1 o 2 & 227 & 44.5 \\
3 o 4 & & 55.5 \\
\hline Evaluación combinada & 318 & \\
A o B & 91 & 77.8 \\
Co D & & 22.2 \\
\hline Ansiedad con importancia clínica & 78 & \\
Sí & 331 & 19.1 \\
No & & 80.9 \\
\hline Depresión con importancia clínica & 114 & 27.9 \\
Sí & 295 & 72.1 \\
No & & \\
\hline
\end{tabular}

Tabla 3. Asociaciones crudas para ansiedad con importancia clínica.

\begin{tabular}{|l|c|c|}
\hline Variables & OR & IC:95\% \\
\hline Edad entre 40 y 64 años & 1.49 & $0.85-2.61$ \\
\hline Sexo femenino & 1.65 & $1.01-2.71$ \\
\hline Estado civil casado (o unión libre) & 1.14 & $0.51-2.54$ \\
\hline Estrato socioeconómico bajo & 1.64 & $0.89-3.03$ \\
\hline Historia de consumo de cigarrillo & 0.71 & $0.36-1.39$ \\
\hline GOLD 3 o 4 & 1.16 & $0.71-1.90$ \\
\hline Evaluación combinada C o D & 1.89 & $1.10-3.27$ \\
\hline
\end{tabular}

Tabla 4. Asociaciones crudas para depresión con importancia clínica.

\begin{tabular}{|l|c|c|}
\hline Variables & OR & IC: $95 \%$ \\
\hline Edad de 65 años o más & 1.19 & $0.86-2.59$ \\
\hline Sexo femenino & 2.10 & $1.35-3.25$ \\
\hline Estado civil soltero & 1.29 & $0.67-2.49$ \\
\hline Estrato socioeconómico bajo & 1.72 & $1.01-2.92$ \\
\hline Historia de consumo de cigarrillo & 1.41 & $0.71-2.79$ \\
\hline GOLD 3 o 4 & 0.97 & $0.62-1.49$ \\
\hline Evaluación combinada C o D & 1.89 & $1.16-3.10$ \\
\hline
\end{tabular}

dad menores a $20 \%$. Esta estrategia se basa en las recomendaciones de Greenland que considera para el ajuste aquellas variables que muestren valores de $\mathrm{p}$ menores de $20 \%$ y que generen un cambio mayor de $10 \%$ en la asociación principal: para el presente estudio ansiedad o depresión con importancia clínica (31). Se aceptó el ajuste si la prueba de HosmerLemeshow mostró valores de probabilidad superiores a 0.05 (32). Se aceptaron como significativas los valores de $O R$ que mostraron IC95\% que no incluían la unidad. El análisis se realizó en el programa IBM-SPSS, versión 23.0 (33).

\section{Resultados}

Participaron 409 pacientes con edades entre 40 y 102 años, media de 73.0 años ( $\mathrm{DE}=10.2$ años), mediana de 74.0 años (RIC $=65-80$ años). Más detalles de las características demográficas se presentan en la Tabla 1. Las puntuaciones para ansiedad se observaron entre 10 y 34 , media de 16.2 ( $\mathrm{DE}=5.2)$, mediana de $15(\mathrm{RIC}=12-19)$ y las puntuaciones para depresión entre 10 y 32, media de $17.0(\mathrm{DE}=5.3)$, mediana de 16 (RIC=13-21). Las variables clínicas categorizadas se presentan en la Tabla 2.

Las asociaciones para ansiedad y depresión con importancia clínica se muestran en las Tablas 3 y 4 . En el análisis multivariado (ajustado), la ansiedad con importancia clínica mantuvo la asociación significativa a la evaluación combinada C o D (OR=2.08; IC95\% 1.19-3.63) y sexo femenino (OR=1.80; IC95\% 1.10-2.99), prueba de HosmerLemeshow: $\mathrm{chi}^{2}=1.41 ; \mathrm{gl}=2 ; \mathrm{p}=0.49$.

En el análisis multivariado, la depresión con importancia clínica se asoció, después de ajustar, a sexo femenino $(\mathrm{OR}=2.35$; IC95\% 1.50-3.70) y evaluación combinada C o $\mathrm{D}(\mathrm{OR}=2.04$; IC95\% 1.22-3.42). Estrato bajo no se relacionó con depresión con importancia clínica al ajustar por sexo y evaluación combinada (OR=1.65; IC95\% 0.95-2.84). Prueba de Hosmer-Lemeshow: chi $^{2}=4.59 ; \mathrm{gl}=4 ; \mathrm{p}=0.33$.

\section{Discusión}

En el presente estudio se observa que $19.1 \%$ de los pacientes presentan ansiedad y $27.9 \%$ depresión con importancia clínica. La ansiedad y la depresión con importancia clínica se asociaron a sexo femenino y mayor severidad de la EPOC, según la evaluación combinada. Se prefirió usar ansiedad y depresión con importancia clínica en lugar de trastorno de ansiedad o trastorno depresivo porque las escalas no hacen diagnóstico formal, sino que identifican a las personas en alto riesgo de presentar un trastorno. No obstante, se debe tener presente que la escala de Zung para depresión mostró sensibilidad de $95 \%$ y especificidad de $75 \%$ (30).

En esta investigación se observó que $19.1 \%$ presentó ansiedad y $27.9 \%$ depresión con importancia clínica. Estas prevalencias algo superiores a lo observado en estudios previos en España, donde observaron prevalencia de ansiedad entre 15.2 y $19.1 \%$ y de depresión entre 9.8 y $14 \%$ con entrevista clínica $(10,11)$. Así mismo, en Colombia se observó 
que $5.8 \%$ de los pacientes informó antecedente de trastorno depresivo (12). Posiblemente la diferencia guarde relación con la forma de evaluación de los síntomas. La prevalencia de trastorno disminuye en forma importante cuando se evalúa con entrevista clínica (34). La prevalencia de síntomas emocionales es superior en los pacientes con enfermedades en situación de cronicidad, por ejemplo, Matte et al. llevaron a cabo una revisión sistemática y metaanálisis y observaron que la prevalencia de depresión en la EPOC fue de $27.1 \%$ (IC95\% 25.9-28.3) y en los controles de $10.0 \%$ (IC95\% 9.2-10.8), es decir, que los pacientes con EPOC mostraron una frecuencia tres veces mayor de síntomas depresivos (OR=3.7; IC95\% 2.4-5.9) (9).

En el estudio actual se observó que la ansiedad y la depresión con importancia clínica se presentaron con mayor frecuencia en mujeres. Esta observación es consistente con la mayoría de las investigaciones que muestran que los síntomas ansiosos y depresivos se identifican más frecuentemente en mujeres, ya sea por una predisposición biológica (34) o por factores de género, sociales y culturales que facilitan la manifestación de los síntomas $(35,36)$.

En este análisis se halló que el estadio GOLD no se asoció a la presencia de síntomas de ansiedad y depresión con importancia clínica. Esta observación es consistente con otro estudio que mostró falta de asociación entre la función pulmonar y la presencia de ansiedad o depresión (10). Sin embargo, Gunawan et al. observaron en 98 pacientes ambulatorios con EPOC en Indonesia, que el porcentaje del volumen espiratorio forzado el primer segundo (FEV1) previsto mostró una correlación moderada con síntomas depresivos, medidos con la escala CES-D $(r=-0.43$; $p<0.001)$ (37).

En el estudio se observó que la historia de consumo de cigarrillo fue independiente de la presencia de ansiedad o depresión con importancia clínica. Este dato es similar a lo informado por Pascal et al. quienes informaron que la historia de tabaquismo o el consumo actual del mismo no guardó relación con el diagnóstico de ansiedad o depresión (15). Esta observación sugiere que el consumo de cigarrillo no es una estrategia para afrontar los síntomas de ansiedad y depresión en pacientes con EPOC como se observa en la población general $(22,23)$. Igualmente se debe considerar un aspecto técnico, el efecto techo es decir, que es difícil observar diferencias cuando la frecuencia de una característica es cercana a $100 \%$ en una muestra particular. En consecuencia, dada la alta prevalencia de consumo de cigarrillo en pacientes con EPOC puede ser difícil establecer una clara diferencia basados en esta variable. Se necesitan estudios con una muestra mucho más amplia (25). Es necesario evaluar rutinariamente síntomas de ansiedad y depresión en pacientes con EPOC. Lamentablemente, estos síntomas no se exploran regularmente porque se considera que son normativos o el paciente no los informa espontáneamente para evitar el estigma-discriminación relacionado con los trastornos mentales (3). En este grupo de pacientes se diagnostican menos los trastornos de ansiedad o depresión y, en consecuencia no reciben el tratamiento médico indicado $(6,14,18,37,38)$.

Este estudio tiene la fortaleza de evaluar síntomas de ansiedad y depresión en una muestra grande de pacientes ambulatorios con diagnóstico de EPOC en Colombia; sin embargo, tiene la limitación propia de los estudios transversales que limitan conocer la dirección de la asociación (39). De la misma manera se deben mirar con precaución las asociaciones para ansiedad dado que no se alcanzó el número de casos para el nivel de confianza y el poder prestablecido (26). No obstante, es necesario tener presente que el tamaño global de la muestra duplicó la muestra mínima y se observaron intervalos de confianza lo suficientemente estrechos (40). La evaluación de ansiedad y depresión con importancia clínica se realizó con escalas de medición y no con entrevista clínica. Pero es necesario considerar los hallazgos dada la sensibilidad y la especificidad que han mostrado las escalas de Zung en población colombiana (29). Nuevos estudios deben evaluar la prevalencia de ansiedad y depresión basados en una entrevista clínica y el mejor manejo farmacológico y psicoterapéutico para los pacientes con EPOC que presentan comorbilidad con ansiedad o depresión.

Se concluye que la prevalencia de ansiedad y depresión con importancia clínica es alta en pacientes con EPOC. La ansiedad y la depresión están asociadas al sexo femenino y a mayor gravedad de la EPOC. Es necesario evaluar la ansiedad y la depresión en todos los pacientes con EPOC seguidos en consulta externa. Se necesitan estudios que evalúen con entrevista clínica la presencia de trastornos de ansiedad y depresivos.

\section{Referencias}

1. Nici L, Donner C, Wouters E, Zuwallack R, Ambrosino N, Bourbeau J, et al American thoracic society/European respiratory society statement on pulmonary rehabilitation. Am J Respir Crit Care Med. 2006;173(12):1390-413.

2. Caballero A, Torres-Duque CA, Jaramillo C, Bolívar F, Sanabria F, Osorio P, et al. Prevalence of COPD in five Colombian cities situated at low, medium, and high altitude (PREPOCOL study). Chest. 2008;133(2):343-9.

3. DeJean D, Giacomini M, Vanstone M, Brundisini F. Patient experiences of depression and anxiety with chronic disease: a systematic review and qualitative meta-synthesis. Ontar Health Techn Assess Serie. 2013;13(16):1.

4. Roncero C. Ansiedad y depresión en el paciente con EPOC. Monogr Arch Bronconeumol. 2017; 3(5):129-32.

5. Zhu B, Wang Y, Ming J, Chen W, Zhang L. Disease burden of COPD in China: a systematic review. Int J Chron Obstr Pulmon Dis. 2018;13:1353.

6. MaurerJ, Rebbapragada V, Borson S, Goldstein R, Kunik ME, Yohannes AM, et al. Anxiety and depression in COPD: current understanding, unanswered questions, and research needs. Chest. 2008;134(4):43S-56.

7. Van Ede L, Yzermans CJ, Brouwer HJ. Prevalence of depression in patients with chronic obstructive pulmonary disease: a systematic review. Thorax. 1999;54(8):688-92.

8. Panagioti M, Scott C, Blakemore A, Coventry PA. Overview of the -prevalence, impact, and management of depression and anxiety in chronic obstructive pulmonary disease. Int J Chron Obstruct Pulmon Dis. 2014;9:1289-309.

9. Matte DL, Pizzichini MM, Hoepers AT, DiazAP, Karloh M, Dias M, et al. Prevalence of depression in COPD: a systematic review and meta-analysis of controlled studies. Respir Med. 2016;117:154-61

10. Arnedillo A, Cordero P, Alfageme I, Lopez-Campos JL, Alcazar B, Casas F. Prevalencia de comorbilidades en pacientes con EPOC. Rev Esp Patol Torac. 2013;25(2):117-24.

11. González MV. Ansiedad y depresión en la EPOC: Prevalencia en una muestra española e impacto sobre la calidad de vida de los pacientes. Granada: Universidad 
de Granada; 2015.

12. Pedrozo-Pupo JC, Campo-Arias A, De La Torre H. Prevalence of comorbidity in patients with chronic obstructive pulmonary disease. Duazary, 2018;15(3):27380 .

13. Atlantis E, Fahey P, Cochrane B, Smith $\mathbf{S}$. Bidirectional associations between clinically relevant depression or anxiety and COPD: a systematic review and meta-analysis. Chest. 2013;144(3):766-77.

14. Miravitlles M, Ribera A. Understanding the impact of symptoms on the burden of COPD. Respir Res. 2017;18(1):67.

15. Pascal OI, Trofor AC, Lotrean LM, Filipeanu D, Trofor L. Depression, anxiety and panic disorders in chronic obstructive pulmonary disease patients: correlations with tobacco use, disease severity and quality of life.Tob Induc Dis. 2017;15(1):23.

16. Blakemore A, Dickens C, Guthrie E, Bower P, Kontopantelis E, Afzal C, et al. Depression and anxiety predict health-related quality of life in chronic obstructive pulmonary disease: systematic review and meta-analysis. Int J Chron Obstruct Pulmon Dis. 2014;9:501.

17. Lecheler L, Richter M, Franzen DP, Rampini SK, Cheetham M, Jenewein $\mathbf{J}$, et al. The frequent and underrecognised co-occurrence of acute exacerbated COPD and depression warrants screening: a systematic review. Eur Respir Rev. 2017;26(144): 170026.

18. Pooler A, Beech R. Examining the relationship between anxiety and depression and exacerbations of COPD which result in hospital admission: a systematic review. Int J Chron Obstruct Pulmon Dis. 2014;9:315.

19. Bolton JM, Walld R, Chateau D, Finlayson G, Sareen J. Risk of suicide and suicide attempts associated with physical disorders: a population-based, balancing score-matched analysis. Psychol Med. 2015;45(3):495-504

20. Goodwin RD. Is COPD associated with suicide behavior? J Psychiatr Res. 2011;45(9):1269-71.

21. Silva JLR, Barreto M, de Sousa K, da Silva C, da Silva L, Rabahi MF. COPD Assessment Test (CAT) score as a predictor of major depression among subjects with chronic obstructive pulmonary disease and mild hypoxemia: a case-control study. BMC Pulmon Med. 2014;14(1):186.

22. Fluharty M, Taylor AE, Grabski M, Munafò MR. The association of cigarette smoking with depression and anxiety: a systematic review. Nicotine Tob Res. 2017;19(1):3-13

23. Goodwin RD, Wall MM, Garey L, Zvolensky MJ, Dierker L, Galea S, et al. Depression among current, former, and never smokers from 2005 to 2013: The hidden role of disparities in depression in the ongoing tobacco epidemic. Drug Alcohol Depend. 2017;173:191-9.

24. Ministerio de Salud de Colombia. Resolución 008430 por la cual se establecen las normas científicas, técnicas y administrativas para la investigación en salud. Bogotá; 1993

25. Gómez C, Sánchez R. Cálculo del tamaño de la muestra en psiquiatría y salud mental:(principios básicos para su estimación). Rev Colomb Psiquiatr.
1998;27(2):131-42.

26. Díaz S, Pita S. Cálculo del tamaño muestral en estudios de casos y controles. Cad Aten Primaria. 2002;9:148-150.

27. Katz MH. Multivariable analysis. Second edition. Cambridge: Cambridge University Press; 2006.

28. Global Initiative for Chronic Obstructive Lung Disease. Global strategy for the diagnosis, management and prevention of chronic obstructive pulmonary disease (2020 Report). Disponible en: https://goldcopd.org/gold-reports/ (Fecha de acceso: 01-10-2020).

29. De la Ossa S, Martínez Y, Herazo E, Campo A. Estudio de la consistencia interna y de la estructura factorial de tres versiones de la escala de Zung para ansiedad. Colomb Med. 2009;40(1):71-7.

30. Campo A, Díaz LA, Rueda GE. Validez de la escala breve de Zung para tamizaje del episodio depresivo mayor en la población general de Bucaramanga, Colombia Biomédica. 2006;26(3):415-23.

31. Greenland S. Modeling and variable selection in epidemiologic analysis. AmJ Public Health. 1989;79(3):340-9.

32. Hosmer DW, Taber $S$, Lemeshow $S$. The importance of assessing the fit of logistic regression models: a case study. Am J Public Health. 1991;81(12):1630-5.

33. IBM-SPSS 23.0. Chicago: SPSS. Inc.; 2015.

34. Aboraya A, Nasrallah H, Muvvala S, El-Missiry A, Mansour H, Hill C, et al. The Standard for Clinicians' Interview in Psychiatry (SCIP): A clinicianadministered tool with categorical, dimensional, and numeric output-Conceptual development, design, and description of the SCIP. Innov Clin Neurosci. 2016;13(56).31-77.

35. Seeman MV. Psychopathology in women and men: focus on female hormones. Am J Psychiatry. 154(12):1641-7.

36. Etienne CF. Addressing masculinity and men's health to advance universal health and gender equality (editorial). Rev Panam Salud Pública. 2019;42:196.

37. Gunawan H, Hanum H, Abidin A, Hanida W. Relationship between depression with FEV1 percent predicted and BODE index in chronic obstructive pulmonary disease. Conference Series: Earth and Environmental Sciences. 2018;125(1):012149.

38. Coventry PA, Hays R, Dickens C, Bundy C, Garrett C, Cherrington A, et al Talking about depression: a qualitative study of barriers to managing depression in people with long term conditions in primary care. BMC Fam Pract. 2011;12(1):10.

39. Coventry PA, Bower P, Keyworth C, Kenning C, Knopp J, Garrett C, et al. The effect of complex interventions on depression and anxiety in chronic obstructive pulmonary disease: systematic review and meta-analysis. Plos One. 2013;8(4):e60532.

40. Calvache JA, Noguera MD. Estimaciones puntuales e intervalos de confianza: resumen de los resultados de investigación. Rev Fac Cienc Salud Univ Cauca. 2007;9(3):57-59. 